\title{
Der Betreuungsunterhalt: Eine Bestandesaufnahme zwei Jahre nach dem Inkrafttreten
}

\author{
Marga Burri *
}

Das Bundesgericht hat seit Inkrafttreten des neuen Kindesunterhaltsrechts am 1. Januar 2017 zwei Leitentscheide dazu gefällt. Seitdem ist klar: Für die Berechnung des Betreuungsunterhalts soll einzig die Lebenshaltungskostenmethode verwendet werden. Der Betreuungsunterhalt bemisst sich folglich nach der Differenz zwischen den Lebenshaltungskosten des betreuenden Elternteils (Bedarfsseite) und dessen Einkommen. Ein Methodenpluralismus wird nicht mehr geduldet. Abgestuft wird der Anspruch auf Betreuungsunterhalt neuerdings nach der Richtlinie des sogenannten Schulstufenmodells. Der vorliegende Beitrag versucht einen Überblick über die bisher erfolgte bundesgerichtliche Rechtsprechung zu verschaffen. Nicht alle Fragen, die sich im Zusammenhang mit dem Betreuungsunterhalt stellen, sind bereits geklärt worden. Nachfolgend wird aufgezeigt, auf welchem Stand die aktuelle Rechtslage ist.

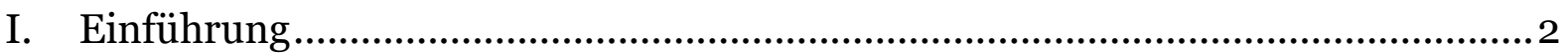

II. Der Lebenshaltungskostenansatz als Berechnungsmethode................................ 3

1. Die bundesgerichtliche Rechtsprechung ...................................................... 3

2. Divergierende praktische Umsetzung .....................................................6

3. Die zu berücksichtigenden Bedarfspositionen der Lebenshaltungskosten .......6

4. Die Einkommensseite...................................................................... 7

III. Die Dauer des Betreuungsunterhalts: Schulstufenmodell....................................8

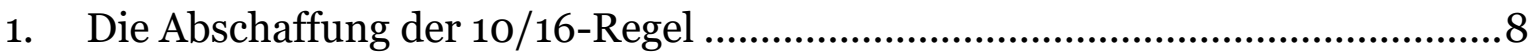

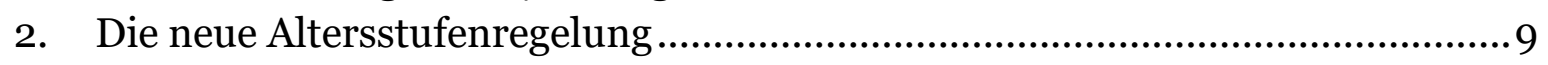

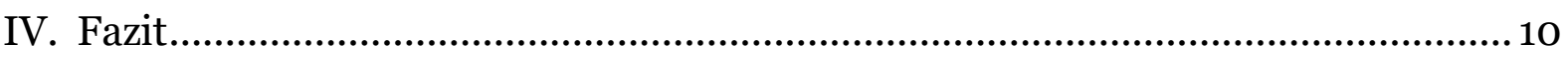

Zitiervorschlag: Marga Burri, Der Betreuungsunterhalt: Eine Bestandesaufnahme zwei Jahre nach dem Inkrafttreten, in: sui-generis 2019, S. 1

URL: $\quad$ sui-generis.ch/88

DOI: $\quad$ https://doi.org/10.21257/sg.88

* Marga Burri (marga.burri@unibas.ch), MLaw, ist wissenschaftliche Assistentin und Doktorandin bei Prof. Dr. Thomas Sutter-Somm, Universität Basel, Juristische Fakultät, Lehrstuhl für Zivil- und Zivilprozessrecht. Der vorliegende Beitrag ist im Rahmen dieser Anstellung verfasst worden. Herzlichster Dank für die kritische Durchsicht des Manuskripts gebührt Frau Meret Rehmann, Rechtsanwältin, Frau Claudine Schär, MLaw, sowie Frau Irina Trutmann, MLaw. 


\section{Einführung}

1 Dem Kind steht seit dem 1. Januar 2017 ein gesetzlicher Anspruch auf Betreuung zu (Art. 276 Abs. 2, Art. 285 Abs. 2 ZGB1). Die Eltern haben gemeinsam sicherzustellen, dass diese Betreuung tatsächlich gewährleistet werden kann. ${ }^{2}$ Das bezweckt der Betreuungsunterhalt als dritte Kategorie des Kindesunterhalts. 3 Anders als der Naturalbedarf deckt der Betreuungsunterhalt nicht die eigentliche Pflege und Erziehung, sondern deren finanziellen Auswirkungen.4 Der Naturalunterhalt darf bei der finanziellen Unterhaltsberechnung nicht vergessen gehen. 5 Sämtliche direkte Kosten, inklusive jene für die Drittbetreuung, werden beim Barunterhalt berücksichtigt. ${ }^{6}$ Der Betreuungsunterhalt umfasst dahingegen die sog. indirekten Kosten, die sich aus dem Mindererwerbseinkommen und dem Anstieg unentgeltlicher kinderbedingter Haus- und Familienarbeit (Ersatzkosten) ergeben.7 Unmittelbare

\footnotetext{
Schweizerisches Zivilgesetzbuch vom 10. Dezember 1907, SR 210.

BGE 144 III 377, E. 7.1.2.1 S. 380.

3 Urteil des Bundesgerichts 5A_384/2018 vom 21. September 2018 E. 4.3

4 Marga Burri, Der Betreuungsunterhalt, Zürich 2018, Rn. 22; vgl. Jonas Schweighauser/Diego Stoll, Neues Kindesunterhaltsrecht - Bilanz nach einem Jahr, FamPra.ch 3/2018, S. 632, 634 f. Zur Relativierung durch blosse Abgeltung des familienrechtlichen Existenzminimums, Rn. 13 und kritisierend Diego Stoll/Roland Fankhauser, Nr. 58 Bundesgericht, II. Abteilung, Urteil vom 21. September 2018 i.S. A. gegen B. - 5A_384/2018, FamPra.ch 4/2018, S. 1085.

5 Sabine Aeschlimann/Jonas Schweighauser, in: FamKomm Scheidung, 3. Auflage 2017, Band I, N. $10 \mathrm{zu}$ den allgemeinen Bemerkungen $\mathrm{zu}$ Art. 276-293 ZGB; Jonas Schweighauser/Daniel Bähler, Betreuungsunterhalt - Berechnungsmethoden und andere Fragen, in: Elterliche Sorge, Betreuungsunterhalt, Vorsorgeausgleich und weitere Herausforderungen, 2018, S. 167; Schweighauser/Stoll (Fn. 4), S. 632 f., 636 f.

6 Urteil des Bundesgerichts 5A_384/2018 vom 21. September 2018 E. 4.3; Burri (Fn. 4), Rn. 20.

7 Burri (Fn. 4), Rn. 21.
}

Folge der Betreuung kann unter Umständen sein, dass der betreuende Elternteil seine Lebensunterhaltskosten nicht mehr decken kann. ${ }^{8}$

2 Wie sich die Höhe und Dauer des Betreuungsunterhalts bemessen, ist auch zwei Jahre nach Inkrafttreten des neuen Kindesunterhaltsrechts noch die grosse Streitfrage. ${ }^{9}$ Die beiden bis jetzt gefällten Leitentscheide des Bundesgerichts stellen erste Grundsätze auf: Der Betreuungsunterhalt ist nach dem sog. Lebenshaltungskostenansatz zu berechnen, andere Methoden werden nicht (mehr) geduldet und die Dauer bzw. Abstufung des Unterhalts richtet sich nach den Schulstufen der Kinder. Damit ist eine zuvor vielseitig monierte Rechtsunsicherheit punktuell beseitigt worden. ${ }^{10}$ Doch die Meinungen zu der bisher ergangenen Rechtsprechung sind teilweise kritisch und einige Fragen bleibnach wie vor unbeantwortet. ${ }^{11}$

8 Weitere mittelbare Folgen (die Erschwerung des wirtschaftlichen Fortkommens, verminderte Karrierechancen, Deckungslücken bei Vorsorgegeldern und Sozialversicherungsbeiträgen) werden nach wie vor nur im Rahmen des nach-/ehelichen (ZGB) Unterhalts (Art. 125, 176 Abs. 1 ZGB) berücksichtigt, siehe Urteil des Bundesgerichts 5A_384/2018 vom 21. September 2018 E. 4.8.3; Botschaft zu einer Änderung des Schweizerischen Zivilgesetzbuches (Kindesunterhalt) vom 29. November 2013 (BBl 2014 529), S. 556; Burri (Fn. 4), Rn. 21, Fn. 50; Stephan Hartmann, Betreuungsunterhalt - Überlegungen zur Methode der Unterhaltsbemessung, in: ZBJV 2/2017, S. $90 \mathrm{f}$.

9 Der gesamte, maximal geschuldete Kindesunterhaltsbeitrag wird nach wie vor durch das zu wahrende Existenzminimum des Unterhaltsschuldners absolut plafoniert, siehe Burri (Fn. 4), Rn. 14 und die Kritik daran in Rn. 32.

10 Alexandra Jungo, Das erste Urteil des Bundesgerichts zum Betreuungsunterhalt: das letzte Wort?, in: Jusletter 13. August 2018, Rn. 4.

11 Jungo (Fn. 10), Rn. 13 ff.; Jonas Schweighauser, Kindesunterhalt - in welche Richtung geht die höchstrichterliche Praxis?, in: Jusletter 17. Dezember 2018, Rn. 32 f., 39; Stoll/Fankhauser (Fn. 4), S. 1085. Zu prozessualen Fragen siehe Cordula Lötscher, Prozessführung und Vollstreckung durch die Eltern im 


\section{Der Lebenshaltungskostenansatz als Berechnungsmethode}

3 Gemäss dem Lebenshaltungskostenansatz entspricht der Betreuungsunterhalt der Differenz zwischen den Lebenshaltungskosten des betreuenden Elternteils und dessen Einkommen.12 Diese Methode entspricht gemäss Rechtsprechung und überwiegender Literatur dem von der Botschaft proklamierten Berechnungsmodell. ${ }^{13}$

\section{Die bundesgerichtliche Rechtsprechung}

4 Der Gesetzestext bestimmt keine konkrete Methode zur Berechnung des Betreuungsunterhalts; die Umsetzung wird der Praxis überlassen. ${ }^{14}$ Bei der viel geäusserten Kritik an dieser Tatsache wird teilweise missachtet, dass dies dem System des Kindesunterhalts vor der Revision entspricht. ${ }^{15}$ Unter altem Recht hatte sich ein Methodenpluralismus entwickelt, den das Bundesgericht duldete. 16

Lichte des Betreuungsunterhalts, in: FamPra.ch 3/2017, S. 621 ff.; Eva Senn, Verfahrensrechtliche Streiflichter zu den Revisionen der elterlichen Sorge und des Kindesunterhaltsrechts, in: FamPra.ch 4/2017, S. $971 \mathrm{ff}$.

12 Für die schrittweise Berechnung siehe Burri (Fn. 4), Rn. 50 ff. und die dortigen Literaturhinweise.

13 BGE 144 III 377 E. 7.1.2.2 S. 383; vgl. Alexander Frei/Matthias Kessler/Christian Wyss/Nicola Imhof, Anwaltsrevue 4/2018, S. 152; Hartmann (Fn. 8), S. 95; für eine andere Sichtweise siehe Roland Fankhauser, Der Betreuungsunterhalt zur Spurensuche und -deutung anhand von Materialien, in: Festschrift für Prof. Dr. Thomas Sutter-Somm, 2016, S. 803; Jungo (Fn. 10), Rn. 11 f., wonach auch die Betreuungsquotenmethode den Vorgaben der Botschaft entspricht; so auch Stoll/Fankhauser (Fn. 4), S. 1086.

14 Vgl. BGE 144 III 377 E. 7.1.2.1 S. 380; Botschaft (Fn. 8), S. 539, 552 f., 555, 575; Fankhauser (Fn. 13), S. 802.

15 Vgl. Burri (Fn. 4), Fn. 8, 59; Hartmann (Fn. 8), S. 89, Fn. 20.

16 BGE 140 III 485 E. 3.3 S. 488; BGE 140 III 337 E. 4.2.2 S. 339; Urteil des Bundesgerichts
5 Seit Einführung des Betreuungsunterhalts am 1. Januar 2017 bis zur ersten klärenden, bundesgerichtlichen Rechtsprechung dazu kamen in der kantonalen Praxis vereinfacht betrachtet drei Berechnungsmethoden zur Anwendung. ${ }^{17}$ Am weitesten verbreitet war der Lebenshaltungskostenansatz. ${ }^{18}$ Vereinzelt wurden eine abstrakte ${ }^{19}$ oder eine konkrete ${ }^{20}$ Betreuungsquotenmethode angewandt. Die Betreuungsquotenmethode gründet ebenfalls auf den Lebenshaltungskosten des betreuenden Elternteils. Dessen Einkommen bleibt hingegen unberücksichtigt. ${ }^{21}$ Entscheidend ist stattdessen die Zeit, die für die tatsächliche Betreuung des Kindes aufgewendet wird. ${ }^{22}$ Es han-

5A_384/2018 vom 21.September 2018 E. 4.1; vgl. Hartmann (Fn. 8), S. 99 f., Fn. 76.

17 In sieben Kantonen hatte sich jedoch auf Ebene der zweiten Instanz noch keine klare Rechtsprechung abgezeichnet, siehe Frei/Kessler/Wyss/Imhof (Fn. 13), S. $151 \mathrm{ff} ., 158 \mathrm{f}$. mit einer tabellarischen Übersicht der Berechnungsmethoden aller 26 Kantone (Stand vermutungsweise Anfang 2018).

18 Soweit ersichtlich wendeten die zweitinstanzlichen Gerichte von 15 Kantonen den Lebenshaltungskostenansatz an, darunter die Kantone Aargau, BaselLandschaft, Bern, Freiburg, Solothurn, Thurgau, Waadt und Zürich, siehe BGE 144 III 377 E. 7.1.2.1 S. 381 f.; Urteil des Kantonsgerichts des Kantons Basel-Landschaft 40017240 vom 14. November 2017 E. 2; 40017155 vom 12. September 2017 E. 10.1; Frei/Kessler/Wyss/Imhof (Fn. 13), S. 158 f.; Schweighauser/Stoll (Fn. 4), S. 641; Annette Spycher, Betreuungs- und Vorsorgeunterhalt - Stand der Diskussion und Ausblick, in: Elterliche Sorge, Betreuungsunterhalt, Vorsorgeausgleich und weitere Herausforderungen, 2018, S. 74, 91.

19 In den Kantonen Glarus und St. Gallen, siehe Frei/Kessler/Wyss/Imhof (Fn. 13), S. $158 \mathrm{f}$.

20 In den Kantonen Basel-Stadt, Luzern, Zug, siehe exemplarisch das Urteil des Appellationsgerichts des Kantons Basel-Stadt ZB.2014.44 vom 13. April 2017 E. 5; Frei/Kessler/Wyss/Imhof (Fn. 13), S. $158 \mathrm{f}$.

21 BGE 144 III 377 E. 7.1.2.1 S. 382; Jonas Schweighauser, in: FamKomm Scheidung (Fn. 5), N. 99 zu Art. $285 \mathrm{ZGB}$.

22 Urteil des Appellationsgerichts des Kantons BaselStadt ZB.2014.44 vom 13. April 2017 E. 5.5.1; Alexandra Jungo/Regina Aebi-Müller/Jonas 
delt sich insofern um eine Pauschalisierung, als einer vollumfänglichen Betreuung ein bestimmter Wert zugemessen wird. ${ }^{23}$ Bei einem reduzierteren Betreuungsbedarf bzw. einer niedrigeren Eigenbetreuungsquote vermindern sich die für den Betreuungsunterhalt relevanten Kosten entsprechend. ${ }^{24}$

6 Das Bundesgericht hat sich erstmals am 17. Mai 2018 vertieft mit der Berechnung des Betreuungsunterhalts beschäftigt und sich für die Lebenshaltungskostenmethode ausgesprochen. 25 Im zweiten Leitentscheid vom 21. September 2018 bestätigte das Bundesgericht nicht nur diese Vorgehensweise, sondern setzte sogleich dem bisher verbreiteten Methodenpluralismus ein Ende. ${ }^{26}$

\section{a) BGE 144 III 377: Der erste Leitentscheid}

7 Am 17. Mai 2018 hat das Bundesgericht den ersten, lang ersehnten Leitentscheid zum Betreuungsunterhalt gefällt. ${ }^{27}$ Beim angefochtenen Entscheid des Genfer Cour de Justice handelte es sich um ein Eheschutzverfahren.28 Gegen die vorsorglichen Massnahmen konnte lediglich die Verletzung verfassungsmässiger Rechte

Schweighauser, Der Betreuungsunterhalt, Das Konzept - die Betreuungskosten - die Unterhaltsberechnung, in: FamPra 1/2017, S. $174 \mathrm{f}$.

23 BGE 144 III 377 E. 7.1.2.1 S. 382.

24 Jonas Schweighauser, in: FamKomm Scheidung (Fn. 5), N. 96, 98 zu Art. 285 ZGB.

25 Vgl. Urteil des Bundesgerichts 5A_454/2017 vom 17. Mai 2018 E. 7.2.2 (unpublizierte Erwägung des BGE 144 III 377).

26 Siehe die Kritik daran bei Schweighauser (Fn. 11), Rn. $32 \mathrm{ff}$.

27 Urteil des Bundesgerichts 5A_454/2017 vom 17. Mai 2018 (auszugsweise publiziert in BGE 144 III 377, übersetzt in Pra 107 (2018) Nr. 104).

28 Urteil des Cour de justice des Kantons Genf C/23428/2015 - ACJC/544/2017 vom 12. Mai 2017. vorgebracht werden (Art. 98 BGG'29). Das fünfköpfige Richtergremium prüfte somit mit eingeschränkter Kognition. ${ }^{30}$ Anlässlich der öffentlichen Urteilsberatung entschied das Bundesgericht mit vier zu eins, die Anwendung der Lebenshaltungskostenmethode zur Berechnung des Betreuungsunterhalts sei im vorliegenden Fall nicht willkürlich. ${ }^{31}$ Im Gegenteil erweise sich der Lebenshaltungskostenansatz als jene Methode, die am ehesten der Absicht des Gesetzgebers entspräche. ${ }^{2}$ Sie sei am besten geeignet, das Ziel zu erreichen, die Einbusse von Erwerbsmöglichkeiten des betreuenden Elternteils aufgrund konkreter Bedürfnisse auszugleichen. 33

\section{b) Die Überprüfung der Berech- nungsmethode auf hinreichend begründete Rüge hin}

8 Kritikern blieb die Hoffnung, dass es nicht der Wille des Bundesgerichts war, mit seinem ersten Leitentscheid zum Betreuungsunterhalt das reine Lebenshaltungskostenmodell als die eine, generell zu geltende Berechnungsmethode vorzuschreiben. 34 In diese Richtung deutete auch ein zwei Wochen später ergangener, nicht publizierter Entscheid betreffend die Abänderung eines Scheidungsurteils:

$2 9 \longdiv { \text { Bundesgesetz über das Bundesgericht (Bundesge- } }$ richtsgesetz) vom 17. Juni 2005, SR 173.110.

30 Urteil des Bundesgerichts 5A_454/2017 vom 17. Mai 2018 E. 2.1 (unveröffentlichte Erwägung des BGE 144 III 377); Jungo (Fn. 10), Rn. 1.

31 Urteil des Bundesgerichts 5A_454/2017 vom 17. Mai 2018 E. 7.2.2 (unpublizierte Erwägung des BGE 144 III 377); 5A_35/2018 vom 31. Mai 2018 E. $4 \cdot 3$.

32 BGE 144 III 377 E. 7.1.2.2 S. 383.

33 BGE 144 III 377 E. 7.1.2.2 S. 384 f.

34 Vgl. Jungo (Fn. 10), Rn. 1, relativierend jedoch Rn. 4; Christian Wyss, Der pauschale Verweis auf Unterhaltstabellen im Entscheid - Fragen zur Begründungspflicht der Gerichte, Anwaltsrevue 9/2018, S. 387 . 
Im vorliegenden Fall hatte das Zuger Obergericht den Betreuungsunterhalt anhand der Betreuungsquotenmethode festgelegt. 35 Da der Sachrichter bei der Festsetzung von Unterhalt «in verschiedener Hinsicht auf sein Ermessen verwiesen ist»36, auferlegte sich das Bundesgericht für dessen Überprüfung Zurückhaltung. 37 Der Beschwerdeführer hatte die Berechnung des Betreuungsunterhalts nach der Betreuungsquotenmethode nicht gerügt. Das Bundesgericht sah daher keinen Anlass, «die Frage der Bemessung des Betreuungsunterhalts von sich aus zu thematisieren, auch wenn ein kürzlich ergangenes Urteil zum Schluss kommt, die Anwendung der so genannten "Lebenshaltungskosten-Methode" sei im konkreten Fall einer Eheschutzstreitigkeit nicht willkürlich gewesen (...).»38

9 So wird denn auch in Verfahren vor dem Bundesgericht der Grundsatz der Rechtsanwendung von Amtes wegen (Art. 106 Abs. 1 BGG) durch die Begründungspflicht im Sinne von Art. 42 Abs. 1 und 2 BGG eingeschränkt.39 Im vorliegenden Fall stimmt daher die Nichtüberprüfung der Berechnungsmethode als Rechtsfrage m.E. mit dieser Rechtsprechung überein. ${ }^{40}$ Die Gel-

$35 \overline{\text { Urteil des Bundesgerichts 5A_35/2018 vom 31. Mai }}$ 2018.

36 Urteil des Bundesgerichts 5A_35/2018 vom 31. Mai 2018 E. 2.

37 Urteil des Bundesgerichts 5A_35/2018 vom 31. Mai 2018 E. 2.

38 Urteil des Bundesgerichts 5A_35/2018 vom 31. Mai 2018 E. 4.3 mit Verweis auf das Urteil des Bundesgerichts 5A_454/2017 vom 17. Mai 2018 E. 7.2 (unpublizierte Erwägung des BGE 144 III 377).

39 BGE 141 V 234 E. 1 S. 236; 140 III 115 E. 2 S. 116; 140 III 86 E. 2 S. 88 f.; Urteil des Bundesgerichts 5A_35/2018 vom 31. Mai 2018 E. 4.3; vgl. Johanna Dormann, in: Basler Kommentar, Bundesgerichtsgesetz, 3. Auflage 2018, N. 1, 5 f. zu Art. 106 BGG.

40 Vgl. Angelo Schwizer/Salvatore Della Valle, Rechtsprechungsupdate zum Unterhaltsrecht, in: AJP 8/2018, S. 1000 f.; anderer Ansicht Wyss (Fn. 34), S. 387 ff., 391. tung des Offizialgrundsatzes für Kinderbelange vor den kantonalen Instanzen gemäss Art. 296 Abs. 3 ZPO41 vermag daran nichts $\mathrm{zu}$ ändern. ${ }^{2} \mathrm{Im}$ bundesgerichtlichen Verfahren gilt grundsätzlich die Dispositionsmaxime gemäss Art. 107 Abs. 1 BGG. Das hat zur Folge, dass die Parteien (bzw. deren Rechtsvertreter) die Berechnung und deren Methode substanziiert hätten rügen müssen, um eine bundesgerichtliche Überprüfung zu erzielen.

\section{c) Das Ende des Methoden- pluralismus}

10 Mit dem zweiten Leitentscheid zum Betreuungsunterhalt statuierte das Bundesgericht, wie der BGE 144 III 377 zu verstehen sei und welche weiteren Grundsätze gelten.43 Es besteht nun kein Zweifel mehr, dass einzig die Lebenshaltungskostenmethode zur Berechnung des Betreuungsunterhalts anzuwenden ist.44 Mit überraschend deutlichen Worten setzte das Bundesgericht dem Methodenpluralismus ein Ende. 45 Es habe sich aufgedrängt, «für die gesamte Schweiz eine einheitliche Methode im Bereich des Unterhaltsrechts zu entwickeln und verbindlich vorzugeben.»46 Dabei bezog sich diese Aussage des Bundesgerichts nicht allein auf den Kindesunterhalt, sondern auf das gesamte Unterhaltsrecht. 47

$41 \overline{\text { Schweizerische Zivilprozessordnung vom } 19 . \text { De- }}$ zember 2008, SR 272.

42 Anderer Ansicht Jungo (Fn. 10), Rn. 3.

43 Urteil des Bundesgerichts 5A_384/2018 vom 21. September 2018 (zur Publikation vorgesehen) E. $4.1,4.4$.

44 Urteil des Bundesgerichts 5A_384/2018 vom 21. September 2018 E. 4.1.

45 Vgl. Schweighauser (Fn. 11), Rn. 32, 34.

46 Urteil des Bundesgerichts 5A_384/2018 vom 21. September 2018 E. 4.1.

47 Schweighauser (Fn. 11), Rn. 34. 


\section{Divergierende praktische Umsetzung}

11 Trotz angestrebter und befürworteter Rechtssicherheit wird die Abkehr vom Methodenpluralismus in der Literatur teils kritisch hinterfragt. 48 Wie gut dieser Grundsatz in der Praxis angesichts der diversen komplexen Konstellationen durchführbar ist, wird sich zeigen. 49

Besondere Bedenken verursachen die kantonalen Unterschiede in der Umsetzung der Lebenshaltungskostenmethode. ${ }^{\circ}$ Solche bestehen gemäss Schweighauser insbesondere «bezüglich der Ermittlung der Lebenshaltungskosten, der Verteilung des Überschusses, der Berücksichtigung des Naturalunterhalts, der Verteilung des Barunterhalts, dem Umgang mit Patchworkkonstellationen».51 Bis anhin gibt es nur wenige höchstrichterliche Ausführungen zur konkreten Berechnung des Betreuungsunterhalts. Das hat auch damit zu tun, dass das Bundesgericht die bisher aufgestellten Leitlinien anhand von Fällen entwickelte, auf welche diese Leitlinien jeweils gar keine konkreten Auswirkungen hatten. ${ }^{2}$

Der Betreuungsunterhalt nach der Lebenshaltungskostenmethode ergibt sich vereinfacht dargestellt aus der Gegenüberstellung zweier rechnerischer Positi-

48 Stoll/Fankhauser (Fn. 4), S. 1085 ff.; ausführlich Schweighauser (Fn. 11), Rn. 35 ff.; begrüssend jedoch Frei/Kessler/Wyss/Imhof (Fn. 13), S. 157.

49 Vgl. Schweighauser (Fn. 11), Rz. 35; Spycher (Fn. 18), S. 70; Stoll/Fankhauser (Fn. 4), S. 1086.

50 Schweighauser (Fn. 11), Rn. 35 f.; Spycher (Fn. 18), S. 92.

51 Schweighauser (Fn. 11), Rn. 36.

52 BGE 144 III 377; Urteil des Bundesgerichts 5A_384/2018 vom 21. September 2018 E. 4.1; Schweighauser (Fn. 11), Rn. 40; Stoll/Fankhauser (Fn. 4), S. 1085. onen: dem Bedarf und dem Einkommen des betreuenden Elternteils. Wie sich diese Grössen genau zusammensetzen bzw. in welchem Umfang sie berücksichtigt werden, kann entscheidender sein als die Berechnungsmethode an sich. 53

\section{Die zu berücksichtigenden Bedarfspositionen der Lebenshaltungskosten}

14 Der Begriff der Lebenshaltungskosten ist durch die Kindesunterhaltsrevision neu eingeführt worden.54 Welche Positionen als Lebenshaltungskosten berücksichtigt werden, hat unmittelbare Auswirkungen auf die Höhe des Betreuungsunterhalts. Gemäss Bundesgericht dürfen die Lebenshaltungskosten den Betrag nicht übersteigen, der für den betreuenden Elternteil notwendig ist, um die Betreuung finanziell $\mathrm{zu}$ ermöglichen.55 Luxuriöse Aufwendungen sind nicht vom Zweck des Betreuungsunterhalts erfasst und somit ausser Acht zu lassen. ${ }^{6}$ Als Grundlage ist auf das familienrechtliche Existenzminimum des betreuenden Elternteils abzustellen, da es um längerfristig geschuldete Unterhaltsbeiträge geht.57 Das be-

53 Mit konkreten Beispielen Spycher (Fn. 18), S. 81 ff., 92.

54 Schweighauser/Stoll (Fn. 4), S. 635.

55 BGE 144 III 377 E. 7.1.4 S. 386 mit Verweis auf Patrick Stoudmann, Le nouveau droit de l'entretien de l'enfant: Ce qui change et ce qui reste, in: Zeitschrift für Kindes- und Erwachsenenschutz (RMA/ZKE) 6/2018, S. 432.

56 BGE 144 III 377 E. 7.1.4 S. 386. Ein luxuriöser Lebensstil kann im Rahmen des Barbedarfs und - bei (ehemals) verheirateten Eltern - der Überschusszuteilung berücksichtigt werden, vgl. Hartmann (Fn. 8), S. 112 f.; Schweighauser/Stoll (Fn. 4), S. $641 \mathrm{f}$.

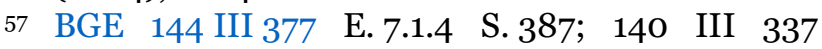
E. 4.2.2 S. 339. Allfällige Unterhaltsbeiträge an Kinder aus anderen Beziehungen werden nicht miterfasst, siehe Hartmann (Fn. 8), S. 98, Fn. 67 mit weiteren Literaturhinweisen. 
treibungsrechtliche Existenzminimum ${ }^{8}$ umfasst nur gerade das Mindestbenötigte während der Dauer des Zwangsvollstreckungsverfahren und ist daher um die familienrechtlichen Zuschläge zu erweitern.59 Für die Erweiterung ist nicht auf Pauschalbeträge abzustellen.60 Was alles berücksichtigt wird, kann sich je nach Einzelfall und - bedauerlicherweise kantonaler Praxis stark unterscheiden. Namentlich die Versicherungen (VVGPrämien, Hausrat- und Haftpflichtversicherungen) und Kommunikationskosten (Fernsehen, Telefon, Internet etc.) können mitumfasst werden. ${ }^{61}$ Bei knappen Verhältnissen sind gemäss Bundesgericht allerdings weder die Steuern, noch die Krankenzusatzversicherungsprämien miteinzubeziehen. ${ }^{62}$ Das führt dazu, dass oftmals überhaupt keine Zuschläge berücksichtigt werden. ${ }^{63}$

Der Pauschalbetrag des betreibungsrechtlichen Existenzminimums (Notbedarf im Sinne von Art. 93 SchKG, Bundesgesetz über Schuldbetreibung und Konkurs vom 11. April 1889, SR 81.1) ergibt sich aus den jeweiligen kantonalen Richtlinien, die in 20 Kantonen jenen der Konferenz der Betreibungs- und Konkursbeamten der Schweiz vom 1. Juli 2009 entsprechen. Nur die Kantone Aargau, Schwyz, Solothurn, St. Gallen und Zürich haben leicht abweichende (bzw. etwas ausführlichere) Richtlinien aufgestellt, siehe Mario Roncoroni, Der Betreibungsalltag - vom Zahlungsbefehl zum Verlustschein, 2011, S. 57. Das Bundesgericht hat die kantonal unterschiedliche Handhabung durch gewisse Grundsätze konkretisiert. So sind beispielsweise Steuern als Auslagen nicht zu berücksichtigen, siehe BGE 140 III 337 E. 4. 2 ff.; BGE 126 III 89 E. 3b S. 92 f.

59 BGE 144 III 377 E. 7.1.4 S. 387.

60 BGE 144 III 377 E. 7.1.4 S. 387; Botschaft (Fn. 8), S. 576; vgl. jedoch Schwizer/Della Valle (Fn. 40), S. 1001.

61 Vgl. Schweighauser, in: FamKomm Scheidung (Fn. 5), N. 81 ff. zu Art. 285 ZGB; Schweighauser/Stoll (Fn.4), Fn. 178; vgl. Hartmann (Fn. 8), S. $108 \mathrm{f}$.

62 BGE 140 III 337 E. 4.2.2 S. 339; Urteil des Bundesgerichts 5A_454/2017 vom 17. Mai 2018 E. 7.2.1 (unpublizierte Erwägung des BGE 144 III 377).

63 Vgl. Schweighauser/Stoll (Fn. 4), S. 638.

\section{Die Einkommensseite}

15 Beim Lebenshaltungskostenansatz spielt der Umfang der Betreuung nur mittelbar eine Rolle. Der maximal mögliche Betreuungsunterhalt in Höhe der Lebenshaltungskosten verringert sich (lediglich) um das anrechenbare Einkommen des betreuenden Elternteils. Grundsätzlich ist vom tatsächlich erzielten Nettoeinkommen auszugehen. ${ }^{64}$ Kann der betreuende Elternteil mit einer Teilzeiterwerbstätigkeit seine Lebenshaltungskosten decken, ist kein Betreuungsunterhalt geschuldet. 65

\section{a) Das hypothetische Einkommen}

16 Soweit die Ausgaben das tatsächliche Einkommen übersteigen, kann allenfalls ein hypothetisches Einkommen an dessen Stelle treten. ${ }^{66}$ Dafür müssen zwei Voraussetzungen erfüllt sein: Erstens muss es dem (wirtschaftlich) berechtigten Elternteil zumutbar sein, ein hypothetisches Einkommen zu erzielen. Welche Tätigkeit als zumutbar gilt, ist dabei Rechtsfrage. Zweitens muss die Erzielung des höheren, hypothetischen Einkommens tatsächlich möglich sein, was eine Tatfrage ist. ${ }^{67}$

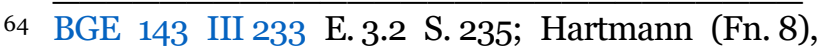
S. 97; Schweighauser, in: FamKomm Scheidung (Fn. 5), N. 127 zu Art. 285 ZGB.

65 Siehe jedoch zur Forderung eines Vorabzugs Rn. 18.

66 Namentlich wenn der betreuende Elternteil einer Teil- oder gar keiner Erwerbstätigkeit nachgeht. Ein potentielles hypothetisches Einkommen ist natürlich auch bei der Erwerbsfähigkeit des Unterhaltsschuldners zu berücksichtigen.

67 BGE 143 III 233 E. 3.2 S. 235; BGE 137 III 118 E. 2.3 S. 120 f.; Urteil des Bundesgerichts 5A_384/2018 vom 21. September 2018 E. 4., 4.9.4. 
17 Wie sich die Erwerbsfähigkeit des potentiell (wirtschaftlich) betreuungsunterhaltsberechtigten Elternteils beurteilt, wirkt sich unmittelbar auf die Dauer des Betreuungsunterhalts aus und wird nachfolgend in diesem Zusammenhalt erläutert. ${ }^{68}$

\section{b) Der Vorabzug}

18 Sinnvoll wäre es, bei der Lebenshaltungskostenmethode einen Teil des vom betreuenden Elternteil erzielten Einkommens nicht zu berücksichtigen. ${ }^{69} \mathrm{Im}$ Sinne eines Vorabzugs bzw. einer Vorabzuteilung eines Teils des Überschusses soll überobligatorische Einkommenstätigkeit abgezogen werden.70 Andernfalls wird der betreuende Elternteil übermässig benachteiligt und der Anreiz für die Erwerbstätigkeit sinkt.71 Besonders bei unverheirateten Eltern führt ein fehlender Vorabzug zu stossenden Ergebnissen, da sie weder Anspruch auf eigenen Unterhalt haben, noch an einem allfälligen Überschuss partizipieren. ${ }^{72}$

19 Die strikte Subtraktionsrechnung bei der reinen Lebenshaltungskostenmethode ohne Vorabzug (gesamtes Einkommen minus Lebenshaltungskosten), kann dazu führen, dass kein oder nur in sehr geringem Umfang Anspruch auf Betreuungsunterhalt besteht.73 Dies obwohl der betreuende Elternteil für den Hauptteil der Betreuungsarbeit aufkommt. Erzielt

68 Siehe III, Rn. 20 ff.

69 Jungo (Fn. 10), Rn. 13, 15; Schweighauser/Bähler (Fn. 5), S. 180; Schweighauser, in: FamKomm Scheidung (Fn. 5), N. 85 ff. zu Art. 285 ZGB; Spycher, (Fn. 18), S. 83, 91.

70 Jungo (Fn. 10), Rn. 13, 15; Spycher, (Fn. 18), S. 83, 91.

71 Jungo (Fn. 10), Rn. 13, 15; Schweighauser/Bähler (Fn. 5), S. 180; Spycher, (Fn. 18), S. 83, 91.

72 Schweighauser/Bähler (Fn. 5), S. 169 f.

73 Schweighauser/Bähler (Fn. 5), S. 169. hingegen der Betreuende mit seinem Erwerbseinkommen einen Überschuss, muss er sich allenfalls neben seiner Betreuungsleistung zusätzlich am Barunterhalt beteiligen. ${ }^{74}$ Der betreuende Elternteil trägt somit einen überproportionalen Teil des Kindesunterhalts. Durch die Korrektur mittels Vorabzug profitiert der betreuende Elternteil vom auch durch seine Erwerbstätigkeit geschaffenen Mehreinkommen. 75

\section{Die Dauer des Betreuungsunter- halts: Schulstufenmodell}

2o Wie lange der Anspruch auf Betreuungsunterhalt in welchem Umfang besteht, bestimmt sich wesentlich danach, ab wann der betreuende Elternteil wieviel Einkommen erzielen kann. Die Revision des Kindesunterhaltsrechts bot Anlass, die während über 20 Jahren geltenden Grundsätze zu überdenken. ${ }^{76}$

\section{Die Abschaffung der 10/16-Regel}

21 Unter altem Recht galt für den nachehelichen Unterhalt die sogenannte 10/16-Regel. Demnach war dem vollumfänglich betreuenden Elternteil (in der Regel die Mutter) mit 10 Jahren des jüngsten Kindes ein Pensum von $50 \%$ und mit 16 Jahren des jüngsten Kindes ein solches von $100 \%$ zuzumuten. Dieser Grundsatz stand immer wieder in der Kritik der Lehre.77 Das Bundesgericht

74 Schweighauser/Bähler (Fn. 5), S. 169 f.

75 Vgl. Schweighauser/Bähler (Fn. 5), S. 170.

76 Botschaft (Fn. 8), S. 578; differenzierend Schweighauser/Stoll (Fn. 4), S. 618, 621, 623 ff.; Stoll/Fankhauser (Fn. 4), S. 1086 f., wonach die 10/16-Regel «nicht unter Berufung auf einen angeblichen gesetzgeberischen Auftrag, sondern weil sich die bisherige Praxis bei genauerem Hinsehen argumentativ als nicht vollends überzeugend erweist» geändert werden soll.

77 Burri (Fn. 4), Rz. 125, 132, Fn. 397 mit weiteren Literaturhinweisen; Schweighauser/Stoll (Fn. 4), S. 618; Schwizer/Della Valle (Fn. 40), S. 1002. 
hatte jedoch konstant daran festgehalten, 78 wenn es auch Ausnahmen und Einzelfallspielraum einräumte.79

Mit dem Leitentscheid vom 21. September 2018 ersetzt das Bundesgericht nun diese 10/16-Regel mit einer neuen Altersstufenregelung. 80

\section{Die neue Altersstufenregelung}

23 Nach einer Übergangsphase oder bei fehlender Vereinbarung kommt als Richtlinie neu die Erwerbsfähigkeit gemäss Schulstufenmodell zur Anwendung: Der hauptsächlich betreuende Elternteil muss ab der obligatorischen Einschulung des jüngsten Kindes grundsätzlich zu $50 \%$, ab dessen Eintritt in die Sekundarstufe zu $80 \%$ und ab seinem vollendeten 16. Lebensjahr zu $100 \%$ erwerbstätig sein. ${ }^{81}$ In den allermeisten Kantonen beginnt die obligatorische Schulzeit mit dem Kindergarteneintritt. Demnach gelten neu die Altersgrenzen von 4/12/16 Jahren. ${ }^{82}$ Je nach Geburtsmonat des Kindes können sich die ersten beiden Stufen um ein Jahr erhöhen. Die für die Abstufung vorausgesetzten Drittbetreuungsmöglichkeiten bedingen einerseits, dass solche effektiv vorhanden sind. Andererseits muss eine tatsächliche Möglichkeit zur Erwerbstätigkeit

$78 \overline{\text { Urteil des Bundesgerichts 5A_98/2016 vom 25. Juni }}$ 2018 E. 3.5; 5A_336/2015 vom 3. März 2016 E. 5.3.

79 Urteil des Bundesgerichts 5A_98/2016 vom 25. Juni 2018 E. 3.5; 5A_957/2014 vom 5. Mai 2015 E. 3.7.2; Schwizer/Della Valle (Fn. 40), S. 1002.

80 Wobei die 10/16-Regel auch im Bereich des nach/ehelichen Unterhalts aufzugeben sei und nicht nur keine Geltung für den Betreuungsunterhalt erlangt, siehe Urteil des Bundesgerichts 5A_384/2018 vom 21. September 2018 E. 4.8.2.

81 Urteil des Bundesgerichts 5A_384/2018 vom 21. September 2018 E. 4.7.6.

82 Urteil des Bundesgerichts 5A_384/2018 vom 21. September 2018 E. 4.7.8; Schweighauser (Fn. 11), Rn. 63; Schweighauser/Stoll (Fn. 4), S. 624; Stoll/Fankhauser (Fn. 4), S. 1088. bestehen, die kompatibel ist mit den (all Semester wechselnden) Schulzeiten und den Drittbetreuungsangeboten. ${ }^{83}$

24 Nach dem Kontinuitätsprinzip rechtfertigt es sich, die bisher gelebte Aufgabenteilung nach der Trennung für eine gewisse Zeit weiterzuführen. ${ }^{84}$ Dieses Prinzip gilt (zivilstandsunabhängig) auch im Bereich des Betreuungsunterhalts, es wird allerdings durch die Altersgrenzen relativiert. 85 Bei der Umsetzung der neuen Abstufung sollen, wie bis anhin schon, nach Möglichkeit grosszügige Übergangsfristen (in Abhängigkeit der konkreten Umstände) gewährt werden. ${ }^{86}$ Aus zureichenden Gründen kann im Einzelfall vom Schulstufenmodell abgewichen werden. ${ }^{87}$ Das Bundesgericht verneint eine Vermutung zugunsten einer möglichst umfassenden Drittbetreuung, stellt allerdings eine diesbezügliche richterliche Prüfungspflicht auf. 88

25 Seit dem Entscheid sind bereits kritische Stimmen laut geworden. An der neuen Altersstufenregelung wird vor allem kritisiert, dass $\mathrm{zu}$ früh schon eine $\mathrm{zu}$ hohe Erwerbstätigkeit verlangt wird.89 Eine $50 \%$-ige Betreuung des Kindes kann nicht mit einer 50 \%-igen Erwerbsfähig-

$83 \overline{\text { Urteil des Bundesgerichts 5A_384/2018 vom }}$ 21. September 2018 E. 4.7.6.

84 Hingegen ist für den strikt zivilstandsunabhängig ausgestalteten Betreuungsunterhalt nicht mehr auf den für die 10/16-Regeln geltende Vertrauensschutzgedanke (Schutz des Vertrauens in den Bestand der Ehe) abzustellen, siehe Urteil des Bundesgerichts 5A_384/2018 vom 21. September 2018 E. 4.4, 4.7.3.

85 Urteil des Bundesgerichts 5A_384/2018 vom 21. September 2018 E. 4.5 f.; vgl. jedoch Rn. 24.

86 Urteil des Bundesgerichts 5A_384/2018 vom 21. September 2018 E. 4.6.

87 Urteil des Bundesgerichts 5A_384/2018 vom 21. September 2018 E. 4.7.9.

88 Urteil des Bundesgerichts 5A_384/2018 vom 21. September 2018 E. 4.7.7.

89 Schweighauser (Fn. 11), Rn. 56; Stoll/Fankhauser (Fn. 4), S. 1088. 
keit des betreuenden Elternteils gleichgesetzt werden. 90 Die Stufe von $80 \%$ Erwerbsfähigkeit ab dem Sekundarschuleintritt des jüngsten Kindes wird in vielen Fällen dazu führen, dass ab dann gar kein Betreuungsunterhalt mehr geschuldet ist. ${ }^{91}$ Der betreuende Elternteil leistet diesfalls Betreuungsarbeit zugunsten beider Eltern, hat diesen aber alleine zu tragen..$^{2} \mathrm{Zu}$ den vom Bundesgericht genannten Übergangsfristen merkt Schweighauser an, dass diese in der Praxis «darauf reduziert werden, wie lange es dauern wird - drei oder sechs Monate? - bis die betreuende Person eine Arbeitsstelle finden kann.»93 Dadurch wird die in der Botschaft getroffene und vom Bundesgericht aufgenommene Annahme relativiert, wonach die Eltern am besten wüssten, welches das für das Kind beste Betreuungsmodell sei.94 Der Kontinuitätsgedanke verliert an Bedeutung.95 Im Streitfall diktiert vielmehr das Gericht, ab wann eine Erwerbstätigkeit in welchem Umfang zuzumuten ist, was eine Drittbetreuung bedingt. 96

\section{Fazit}

26 Nicht nur Juristen haben die Rechtsprechung zum Betreuungsunterhalt mit Spannung erwartet. Das Bundesgericht

$90 \overline{\text { Burri (Fn. 4), Rz. 130; ausführlich Schweighauser }}$ (Fn. 11), Rn. 54 ff.; Annette Spycher, Kindesunterhalt: Rechtliche Grundlagen und praktische Herausforderungen - heute und demnächst, in: FamPra.ch 1/2016， S. 22; Stoll/Fankhauser (Fn. 4), S. 1089.

91 Schweighauser (Fn. 11), Rn. 59.

92 Jungo (Fn. 10), Rn. 13; Schweighauser (Fn. 11), Rn. 59; Stoll/Fankhauser (Fn. 4), S. 1089.

93 Schweighauser (Fn. 11), Rn. 45.

94 Urteil des Bundesgerichts 5A_384/2018 vom 21. September 2018 E. 4.5; vgl. Botschaft (Fn. 8), S. 555, 577; Schweighauser (Fn. 11), Rn. 51.

95 Schweighauser (Fn. 11), Rn. 43; Stoll/Fankhauser (Fn. 4), S. 1087, 1090 f.

96 Vgl. Schweighauser (Fn. 11), Rn. 43, 51. versucht, durch die bisher erfolgten beiden Leitentscheide möglichst klare Leitlinien aufzustellen. Das ist begrüssenswert. Die durch die Änderung des Kindesunterhaltsrechts vielseitig monierte Rechtsunsicherheit soll nach und nach beseitigt werden.

27 Inhaltlich kann die Bestimmung des Lebenshaltungsansatzes als einzig anzuwendende Berechnungsmethode zwar kritisiert werden. Die Lebenshaltungskostenmethode gelangte jedoch in den allermeisten Kantonen schon vor BGE 144 III 377 zur Anwendung. Dennoch kann die nach wie vor kantonal divergierende Umsetzung dieser Methode zu grossen Unterschieden führen. Das ist zu bedauern und widerspricht der Devise des Bundesgerichts, mehr Einheitlichkeit in der ganzen Schweiz zu schaffen. Ob sich die Verabschiedung des Methodenpluralismus bewährt, wird sich anhand der kommenden Entscheide zeigen.

28 Durch die Abstufung des Unterhaltsanspruchs nach dem Schulstufenmodell wird von betreuenden Eltern ein früherer Wiedereinstieg in die Erwerbstätigkeit verlangt. Es ist fragwürdig, ob sich die vorausgesetzten, hohen Erwerbspensen mit dem Familienalltag vertragen.

29 Vor allem beseitigen die Bundesgerichtsurteile damit die vielseitig kritisierte Rechtsunsicherheit. Im Übrigen werden mit den bisher erstellten bundesgerichtlichen Leitlinien die Konsequenzen der Einführung des Betreuungsunterhalts abgeschwächt. Die Anwendung der Lebenshaltungskostenmethode ohne Vorabzug führt in vielen Fällen zu tiefen Betreuungsunterhaltsbeiträgen, und durch das Schulstufenmodell werden schon 
früher höhere Erwerbspensen zugemutet. Betreuungsunterhalt ist während kürzerer Dauer als Barunterhalt geschuldet, insbesondere nur falls und so lange der betreuende Elternteil nicht selbst für seine Lebenshaltungskosten aufkommen kann. 